\title{
Namenregister Nr. 16
}

Ein vollstăndiger oder gekurzter Abdruck der Referate ist ohne Gienehmigung der Redaktion und des Verlages verboten!

\begin{tabular}{|c|c|c|c|c|c|}
\hline 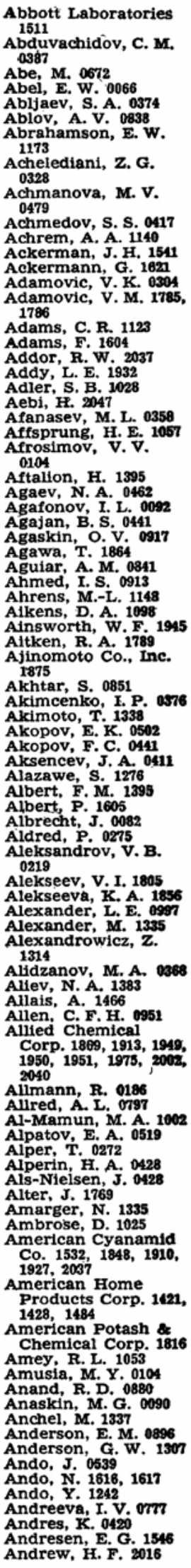 & 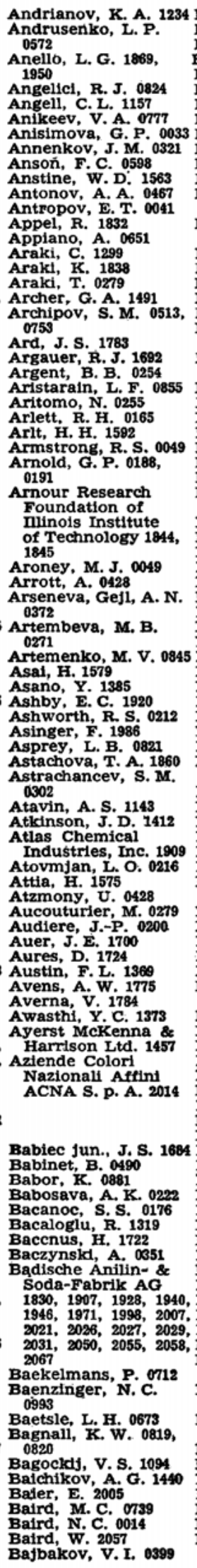 & 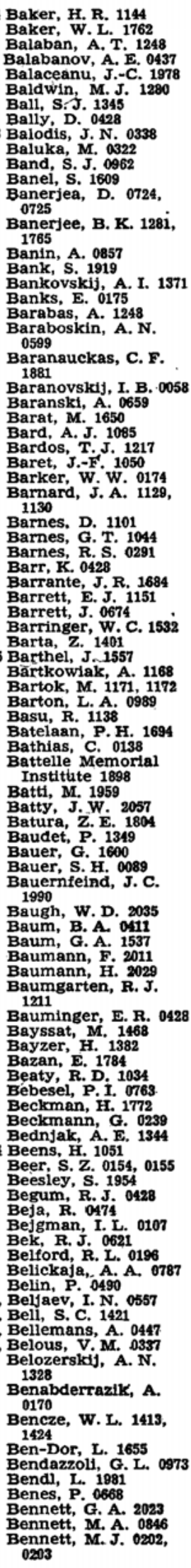 & 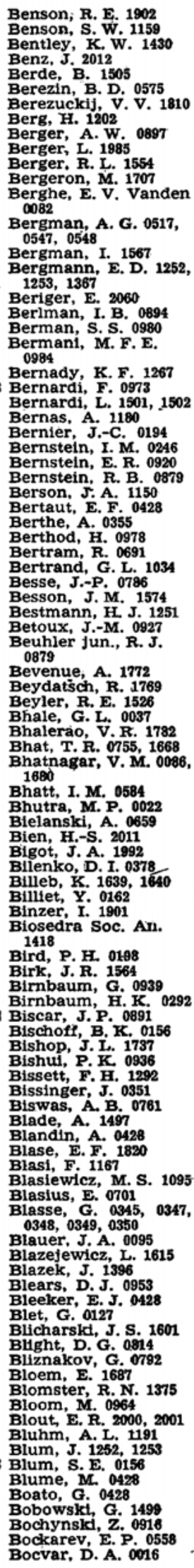 & 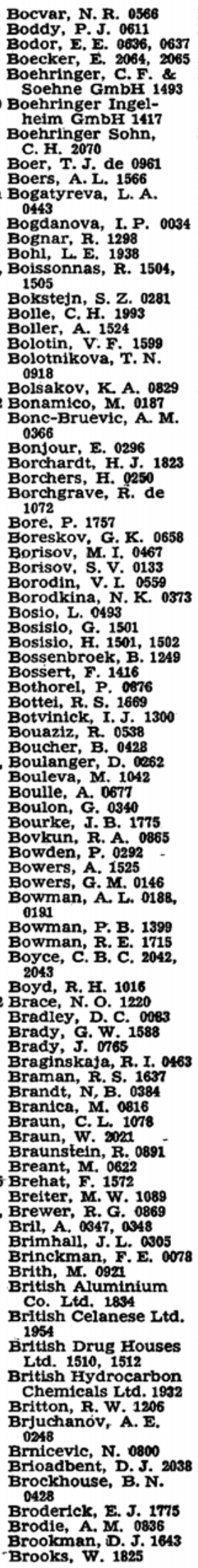 & 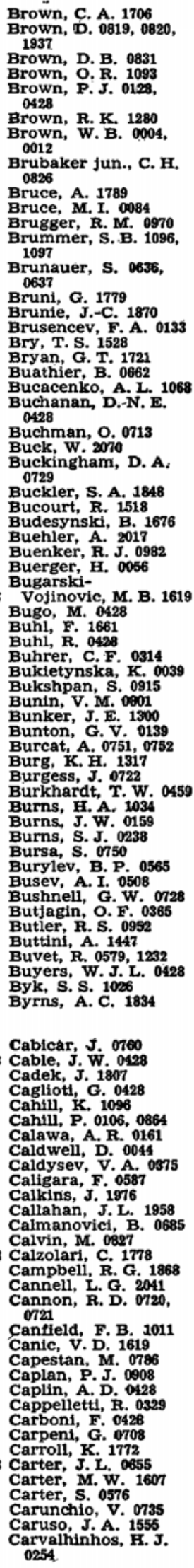 \\
\hline
\end{tabular}




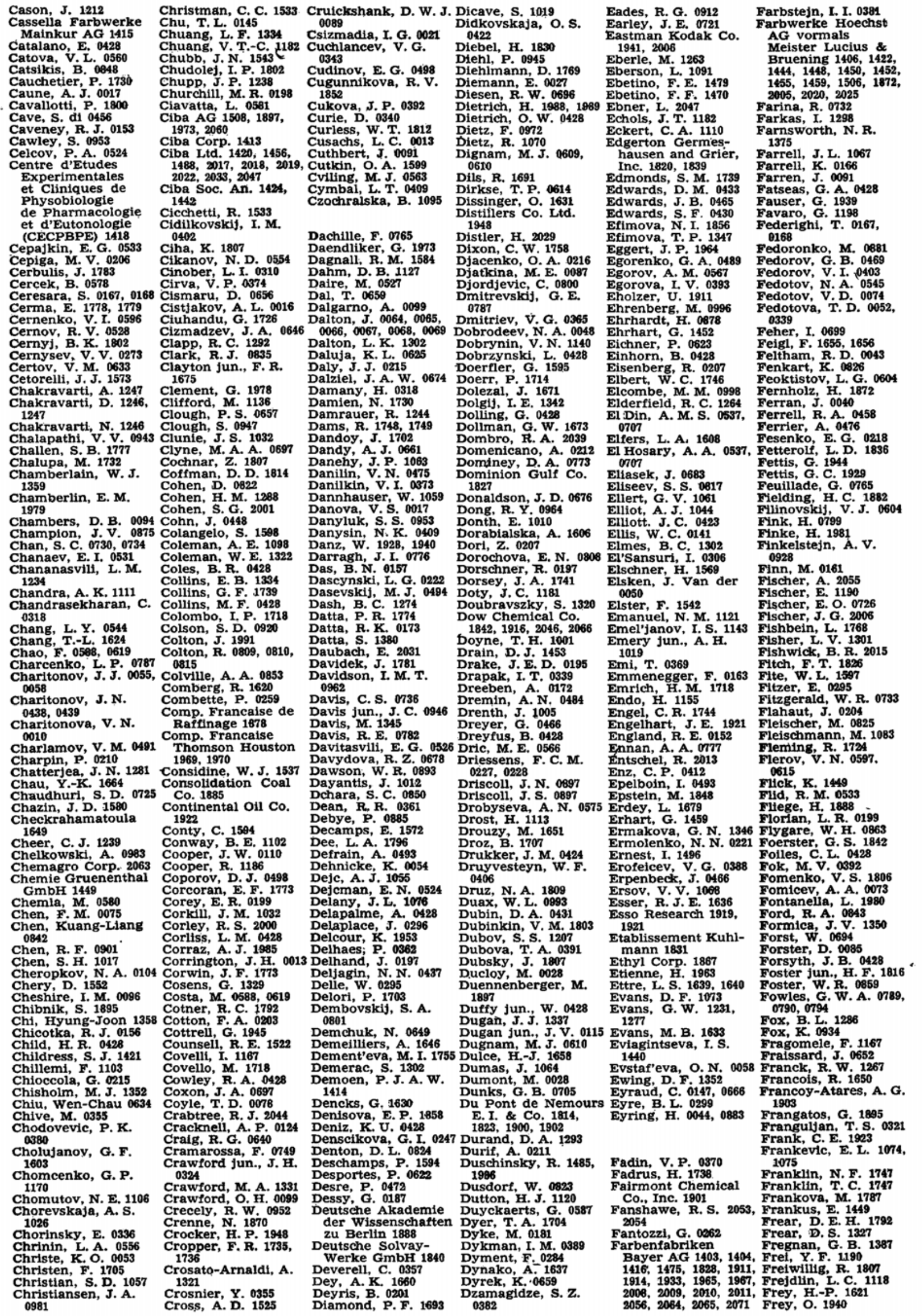




\begin{tabular}{|c|c|c|c|c|c|}
\hline 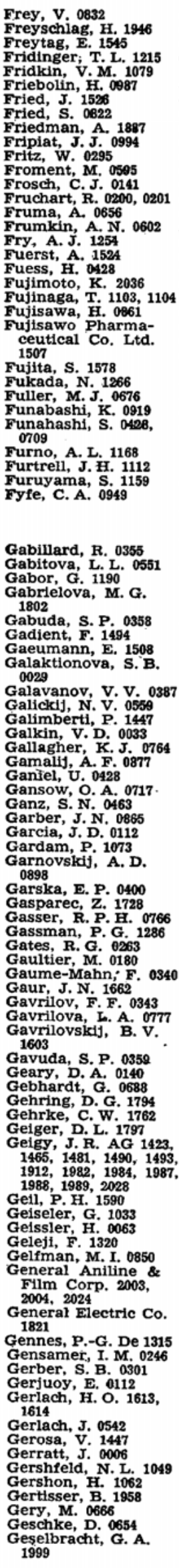 & 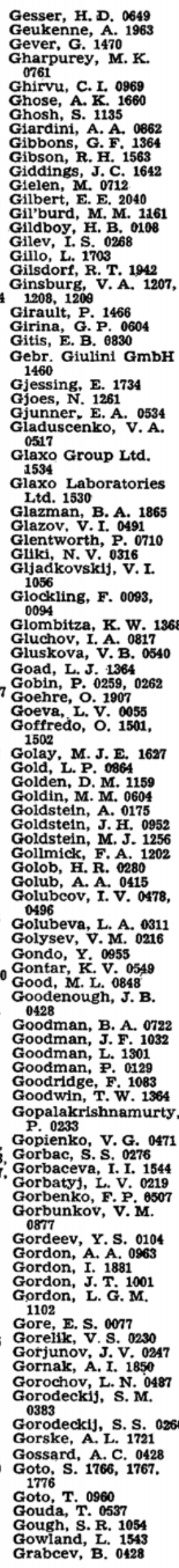 & 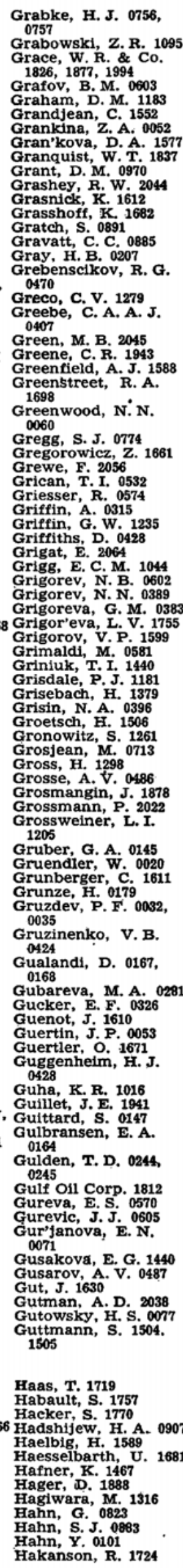 & 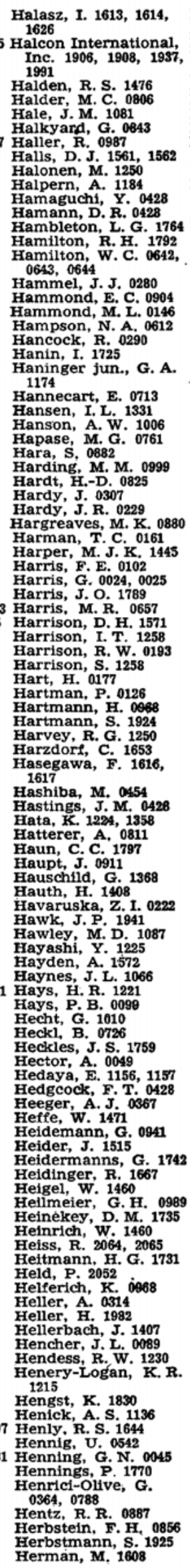 & 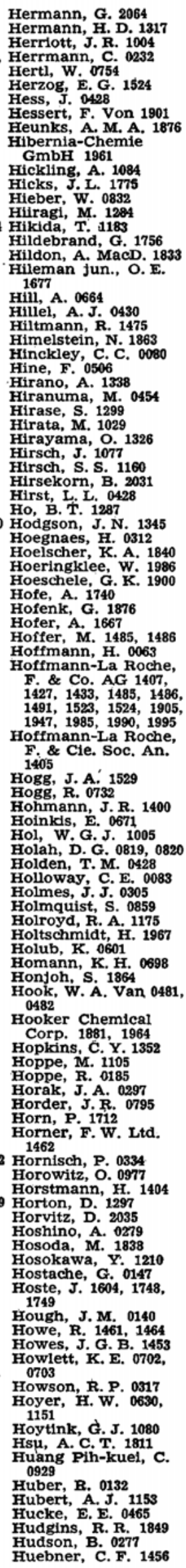 & 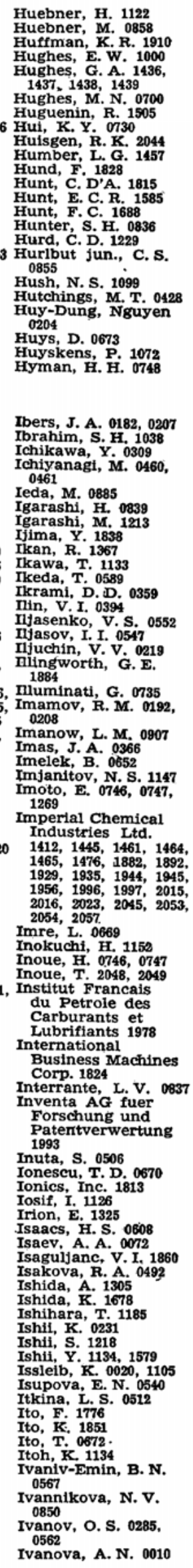 \\
\hline
\end{tabular}


Ivanova, O. M. 1061 Ivanovskij, G. F. 049 Iwakura, Y. 0772 Iyengar, P. K. 0428 Izotov, V. I. 0445

Jablikov, J. v. 0363 Jaccarino, V. 0428 Jachkind, N. D. 0534 Jacimirskd, K. B. 0568

Jackson, L. L. 1327

Jackson, R. 0254

Jacobs, T. 0665

Jahr, K. F. 1559

1463 . $T$.

Jakobi, N. J. 0516, 0530 Kamiya, Y. 1857

Jakobi, N. J. 0516,0530 Kamiya, Y. 1857
Jakobson, G. G. 1278 Kamm, G. 1727

Jakobson, G. G. 1278 Kamm, G. 1727

Jakusev, V. G. 0485

James, B. R. 0737

Jan, R. von 0293

Janev, R. K. 0114

Janin, J. 0340

Jano, I. 1178, 1179

Janssen, G. 1308

Janssen, O. 0919

Janssen Pharma-

ceutica N.V. 141

Japar, S. M. 11

Jarceva, R. D. 0524

Jaroslavskij, M. I.

0424

Jedrzejczak, M. 0750

Jenden, D. J. 172

Jennings, D. A. 0932 ennings,

Jensen,' M. A. 0367;

Jentzsch, D. 1630

Jerchel, D. 1515

Jermyn, M. A. 1291

Jernejcic, M. 1645

Jette, A. N. 0106
Jeweil, J. S. 1297

Jezowska-

Trzebiatowska, B. 0039, 0322

Jha, H. C. 1281

Jindra, J. 0590

Joergensen, C. K. 0002

John, T. L. 0103 Johari, P, 1059

Johnson, C. R. 1239

Johnson, D. P. 1689 0768

Johnson, G. S. 202

Johnson, J.R. 1057

Johnson jun., J. S.

Johnston, K. M. 1697

Jonassen; H. B. 0841

Jones, A. 1196

Jones, A. 1196

Jones, G. G. 1555

Jones, J. K. N. 129

Jones, P. C. 0612

Jones, T. A. 0912

Jones, T. P. 0718

Jones, W. M. 048

Jong, J. de 1974

Jordanov, N. 1752

Jork, H. 1696

Jork, H. 1696
Jortner, J. 0921,1077
Joshi, N. D. 1665

Joshua, S. J. 012

Jounel, B. 0472

Jucker, E. 1494

Juda, W. 1813

Judkovskij, S. I.

Jung, C. J. 1434

Jurd, L. 1915

Jusibova, A. D. 0462

Just, E. 0799
Just, T. 1549

Kanekó, T. 1304

Kaner, E. A. 0408

Kanner, B. 1880

Kaplan, G. B. 1400

Kaplan, I. G. 0434

Karasik; V. R. 0417

Karavaev, G. F. 0375

Karawya, M. S. 179

Karla, P.S. 0400

Karle, L. 0995

Karnaev, N. A. 059

Karpovic, I. A. 0398

Kars, F. A. 1876

0753

Kastler, A. 0046

Kataoka, T. 1312

Katayama, S. 0586

Kathren, R. L. 1551

Kato, H. 0967, 1275

Kato, K. 2048, 2049

Kato, S. 1204

Katsurai,' T. 0150

Katz, E. 1350

Katz, B. 0921

Kauffmann, J.-P.

aul, B. 1365

Kauppila, W. E. 159

Kawami, 1. 1838

Kawanami, J. 1513

Kawanama, Y. 185

Kawano, N. 1282

Kawano, Y. 1772

Kawase, Y. 1260

Kawski, A. 0892

Kazakov, V. K. 0266 0403

B. A. 0872

Kebabcioglu, R. ${ }^{0057}$

Kecin, V. V. 0406

Kecskes, M. B. 1772

Keenan, T. K. 0821

eii, T. 1192

Keith, J. N. 0748

Kelen, G. P. Van de

Keler, E. K. 0540

Keller, B. 0039

Keller, E. 1982

Keller, F. 1300

Keller, M. 1912

Kellogg, R. M. 1261

Kelly, A. W. 1883

Kemnitz, D. A. 1741

Kenson, R. E. 0782

Kepert, D. L. 0807 ,

Keresztesy Jun., J. C.

Kerkhoffs, P. L. 199

Kermarec, J. 0652

Kern-Bausch, L. 032

Kerrigan, J. V. 1815

1154 marthy, $\mathrm{N}$.

0440

Kabas, G. 1237

Kac, I.

Kackurova, I. J. 0938

Kadar, G. 0428

Kadlecova, L. 1657
Kagami, K. 1855
Khetrapal, C. L. 0945 Konishi, H. 0967

Khodair, I. A. 121

Khoobiar, S. 0655

Kiang, A. K. 1374

Kida, Y. 0746, 0747

Kier, L. B. 1279

\& Kigasawa, K. 1284
Kikuchi, K. 0888, 1199

Kikuchi, T. 1185

Kilpatrick, P.J. 1057

Kim, A. M. 1146

Kimiova, i. 1401 Kopylov, N. I. 0546

Kindler, H. 2007

. (1) Korletjanu, L. N. 0838

Kingsbury, P. I. 0226 Kornienko, V. P. 0532 Kinoshita, S. 1339

Kirgey, J. L. 035 rgincev, A. N. Korsunskij, M. I. 04

Kirin, I. S. 0404

Kiriyama, T. 1678 Kirkinsklj, v. A. 0485 Kotova L. P. 0111

Kirss, V. 1975

Kisalei, D. 0466

Riseleva, S. I. 1346

Riselevskij, L. $\mathrm{r}$.

kiskin, S. T. 0281 089

Kitchlew, A. 0076

Kittel, E. 1940

Klaui, H. 1995

Kleb, K.-G. 2008

Kleber, H. 1670

(leiman, R. 171

Kleinman, C. J, 01

Kleinstauber, A. T.

Klevkov, J. v. 0270

Klicka, J. 1798

Klimanek, P. 026

Klimov, A. N. 1347

Klimova, i. A. 1056

Klimstra, P. D. 1522

Klingman, G. E. 186

Kljuev, B. L. 1092

Kloetzscher, I. 1924

Klopman; G. 0693

Klotz, H.-D. 1113
Klusina, T. v. 078

Knackmuss, H.-J. 132

Knight, R. J. 0887

(j)

Knjazeva, N. A. 0055

Knobloch, E. 1397

Knoerenagel, K. 1918

Knopp, J. A. 0903

nowles, A. 1200

Koehler, w. 1033

Koehler, w. C. 0428

Koenig, K.-H. 20

Koenig, W. 1938

macher, A. 1750

Koetzle, T. F. 017

Kohler, P. 1769

Koizumi, M. 0888, 119

Kokot, I. F. 0360

Kokubun, H. 0888

Kolesnikov, v. N.

0041

Kolesova, R. V. 0218

llar, J. 1900

Kollegov, V. F. 1139

Kolobich in , V.

Kolomiec, B. T. 038 0176

Kolotilo, D. M. 0966

Komarov, v. S. 1850

0036

Kometani, T. Y. 0428

Kondow, T. 115

Kondratov, I. J. 190

Koneva, N. A. 0257

Konevskdy, V. S. 033

Konin, K. P. 0386

roninke

Zwavelzuur-

Pabrieken v/h
Ketjen N. v. 1974

Konnov, J. I. 0302

conoshima, M. 1358 Konovalov, E. E. 0505 . 1926 opfmann, G. 0132

Korotaev, A. P. 0257 Korsunov, B. G. 055 Koshcheenko, K. A. Koto

Kovalskij, A. E. 0274 Koyama, K. 1090 Kozyrev, B. M. 1323 Kramarenko, N. L. 0319

Kramlova, M. 1716

Kranz, J. 2026, 2027 13

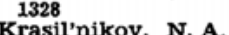

Krasnobaeva, N. 1752 Krause, G. F. 1762, 1763

Krauss, L. 0038

Krauss, M. 0105

Krefeld, M. E. Van

0050

Krejskop, V. N. 0310

Kren, E. 0428

Kricevskij, I. R. 050

Kriegsmann, H. 0059,

Kriegsmann, K. 0063 Krikorian, N. H. 0188, 0191

Kripjakevic, P. I. 0206 Krischer, J. 1712 Krischer, H. 0334 Krishnamurty T. S. G. 0e33,

rishnan, R. 0428

Krochina, A. I. 0294

Kroneberg, H.-G.

1475

Kroneisen, A. 1613,

, N. 0428

Krueger, H. 1630

Kruglov, V. 1. 0396 
Lonza Elektrizitaets-
werke und Chemische Fabriken AG sche

Looker, J. J. 1236

Loomis, B. A. 0301

Lorentz, K. 1713

Lossing, F. P. 1196

Lotti, G. 1784

1903

Love, W. E. 1004

Lovesey, S. W. 0428

Low, G. G. 0428

Lowde, R. D. 0428

Lozgaceva, L. A. 0550

Lu, B. C.-Y. 1031

ucas, J. M. S. 134

Lucchesi, C. A. 1027

Lucken, E. A. C. 0959

Ludwig, H. 0225

Lueck, W. 1600 .

0488

Lueders, G. 0413

Lukas, I. R. 1547

Lukjancuk, S. V. 0928

Lund, H. 1066 . 0358

Lunelli, B. 0937

Lungu, A. M. 0428

Lust, S. 1536

Lutz, H. D. 0062

Lutz, w. L. 1480

Luxinskaja, M. G.

Luznova, M. I. 1383

Lwowskd, w. 119

Lydon, J. E. 0214

Lyne, F. A. 1686

yscov, A. I 0559

Lysenko, N. J. 0410

M. \& T. Chemicals,

Inc. 1537, 1920, 2034
Ma, R. 0895

Mabbs, F. E. 0834 Markert, F. 1940

Maccabee, H. D. 0289 Markillie, J. H. 147

McCarthy, w. J. 157 M Markin, v. S. 0646

McClintock, M. 0932

c. Conaghy jun.

Maccone, S, 2062

McyCord, T. G. 1560

McCoy, H. E. 0166

McCoy jun., H. E.

McDonald, M. P. 1002

McDowell, C. A. 0949, 0954

Mac Ewen, J. D. 179

Co. Ltd, 1480

MCFarlane, I. J. 1378

McFarlane, w. 0361

McGreevy, A. E. 1936

Mach, E. A. 048

Machi, S. 1316

Mach

McIsaac, W. M. 1287

Mack, M. E. 0889

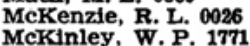

McLachlan, D. s.

MeLamore, w. $\mathbf{M}$.

1489

McLaren, L. 1642

McLeod jun., D. 1157

McLeod, H. A. 1771

McRickard, S. B. 0308

Madaeva, O. S. 1366

Maddock, A. G. 11

Magee, J. L. 0115

Magee, R. J. 2037

Magyar, B. 0784

Mahajan, S. N. 0428

Mahapatra, G. N. 1

Maher, D. M. 0299

Mahmood, A. J. 0710

Mahn, C. 0120, 012

Main, M.

Maillard, P. 0137

Majid, Y. A. 0702

Majzus, Z. K. 1121

Majzarevic, L. A. 0501

Makesina, A. V. 0558

Maki. A. H. 0955

Mamy, J. 0660
. 1854

0522 . 03 Maksimovskij, S. N.

. 007

1361,1370

. F. 0740

cev, A. A. 0042

Malfroid, M. 0712

. U. 0635

alinin, L. v. 0878

Malissa, H. 159

Malkova, T. V. 0568

Malone, C. T. 1758

Maloy, J. 'T. 1085

Malyseva, E. A. 1802

Mamaev, V. P. 1146

Manakov, M. N. 186 randy

Manev, E. 1042

Mani, J.-C. 1177

Mank, V. V. 0360

Mann, R. S. 1117

Mannila, K. D. ${ }^{0061}$

Manson, S. 0110

Mansurova, K. S. 1106

Marangoni, L. 1539

Marazovic, L. 17

Marchand, A. 0362

Marcus, M. F. 1087

Mardix, S. 0269

Margarian, R. A. 125

argarian, R. A. 1259

arinelli, L. 1788

Marini, P. 0679 .

Markillie, J. H. 14

Markina, M. v. 054

Kulov, A. V. 0615

Markovskij, L. J. 0783 Merrifield, R. E. 0890

Markovskij, V. J. 0276 Merz,

Marle, C. 0638 0276 Mescerjakov, A. P.

Marletta, G. P. 177

Marsay, C. 0767

Marsh, H. 0664

Marsigny, $\mathrm{L}$. 0040

Martel, P. 0428

Martin, C. 0211

Martin, H. 2047

115

Martín, J. K. 1602

artin jun., J. M.

1737

0927

. P. P. 0573

artin jun., w. W. 1815

Cartin-Marietta

Martindill, G. H. 116 Martinkova, J. 1340 Martynov, E. D. 028 Martynova, L. L.

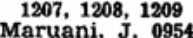
Maruani, J. 0954 Marusin, V. D. 0034 Masik, M. G. 1384 Maskill, R. Or11 Mason, R. 0202 Masoud, A. N. 1375 Masovec, T. V. ${ }^{0308}$ Masson, D. B. 0473

Mastrjukov, V. S.

Mathews, F. S、 013 Mathieu, J.-C. 0472 Mathur, J. N. 0436 Matrai, M. 0235 Matschiner, H, 110 Matsrjukov, v. $\mathbf{s}$.

Matsubara, T. 0425

Matsuda, K. 1324 atsui, M. 0689

atsumoto, $\mathrm{H} .186$

atsuno, S. 0539

Matsutani,'s. 1304

Matsuura, S. 0960

Matsuyama, K. 1862

Matwiyoff, N. A. 008

rauger, R. 1064

May \& Baker Ltd.

ayo, P. de 1337

Mayr, H. H. 1382

Mazeron, P. 171

Mazey, D. J. 0291

Mazzola, V. J. 1267

Mazzucato, U. 1198

Medina, M. V. 1376

Medvedev, A. N. 1207,

120,1209

Medvedev, E. S. 0450

Meer, W. A. 1375

ehl, W. 1081

Melech. B. T. 0480

Melichov, I. V. 0503

Melzer, w. 1586

Menabde, N. E. 0457

Mendelovici, E. 0994

Mendoza, C. 1771

Menees, G. P. 0026

Mentinger, F. 0428

enyhard, N. 0458

zinger, F. 0428

Merck, E., AG 1477 ,

1536
Merck \& Co., Inc.

1458, 1472, 1519, 1

1533, 1873, 1979

Merkel, 1835

(

Meshitsuka, G. 0957

Metayer, M. 0686

Meyer, R. B. 0988

Michaeljan, V. M.

Michaud, P. 1165

Michel, A. 0162, 0200 ,

0201

Michels, H. H. 0102 1343

Mícka, K. 1672

Mickey, J. 0280

Midorikawa, F. 1213

Mikerina, A. L. 1342

0637

Gin, 1155

Milburn, R. M. 074

Inc. 1959

Miller, K. J. 0105

Miller, R. L. 1003

Millhouse, A. H. 042

Milligan, B. 1182, 1231 .

Mills, O. S. 0832

Miloslavsidj, v. K.

Milovanova, S. N.

1342, 1344

Mine, 1857

Minegishi, A. 0957

Minina, N. J. 0384

0899,0900

Minlibaeva, A. N.

1162,1163

Miodownik, A. P.
2025

Mikhail, R. S. 0686 .

Mile, B. 1007

Miles, D. W. 0883

Miles Laboratories,

Miletic, N. 1785, 1786

Miller, E. A. 014

Mioseu, M. 1556

Murakam1, K. 1838

Mironenko, A. P. 0514 Muren, A. P. 1938

Misawa, M. 1339

Misra, C. H. 0844

Misurkin, I. A. 001

Mitra, C. R. 1373

Mitsch, R. A. 1114

Mitsui, H. 1316

Mitsui Kagaku Kogyo

K. K. 2048, 2049

Mittler, A. 1772

Miura, H. 1282

Miyajimá, K. 1695

Miyashiro, J. 1962

Mizusaki, S. 136

Mizushima, M. 0932

Mizutani, T. 2036

Mjasoedov, M. I. 1852

noucek, $\mathrm{K} .1397$

oal-Daire, M. F. Le N. V. Pharmacia 1410

Combinatie voor

Combinatie voor

Industrie 196

Mochammed, C. 0306

Mochosoev, M. V,

Modi, A. P. 1135

Nabara, A. 0872

Moenke, H. 1591, 1593 Nabereznych, V. P.

Moenke-Blanken-

burg, L. 1581

Moghisse, A. A. 1607

. V. 0319

Mohilner, P. R. 0600 Naepfel, H. 0005

Mohr, G. 1536 Nagasawa, N. 1290

Moin, F. B. 116

Moisar, E. 076

Mojzes, B. J. 0391 


\begin{tabular}{|c|c|c|c|c|c|}
\hline 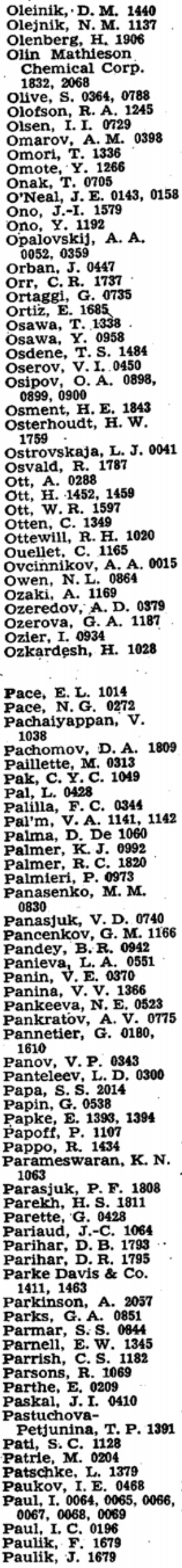 & 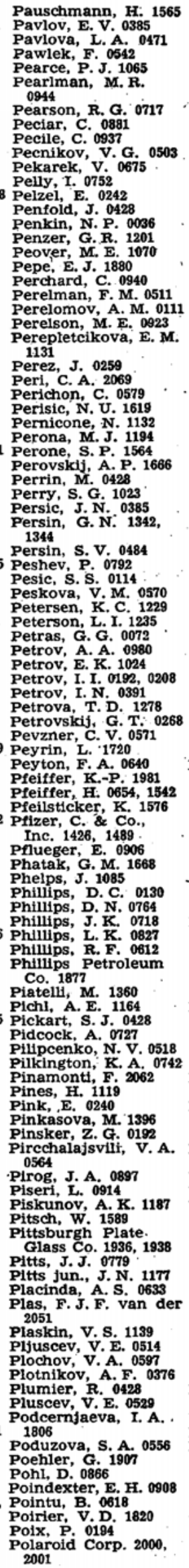 & 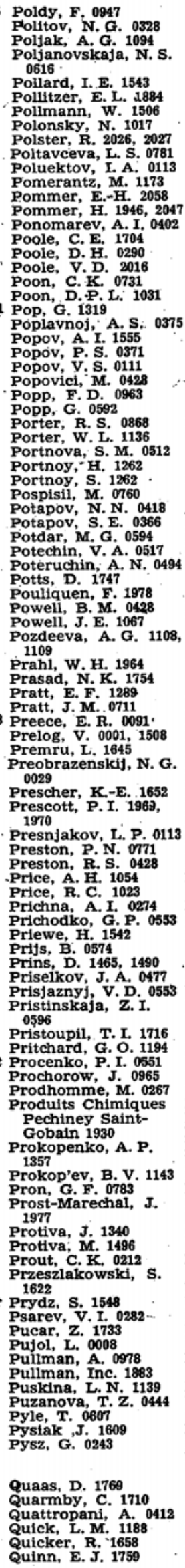 & 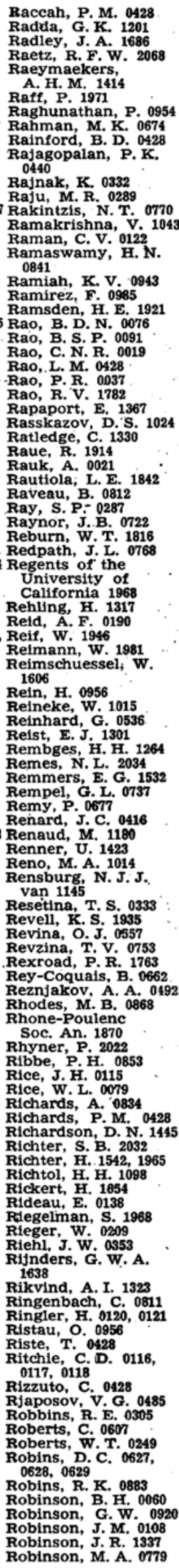 & 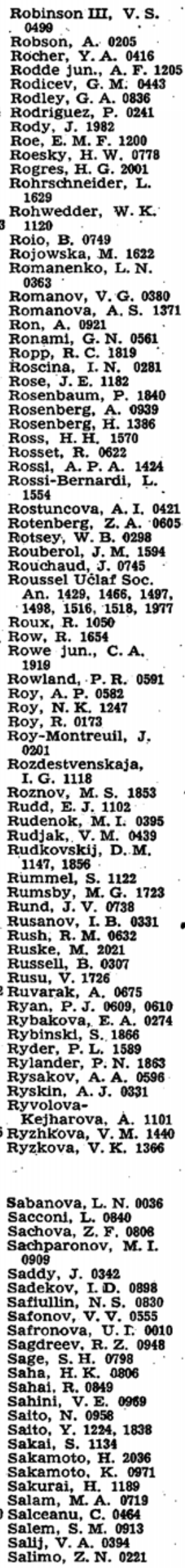 & 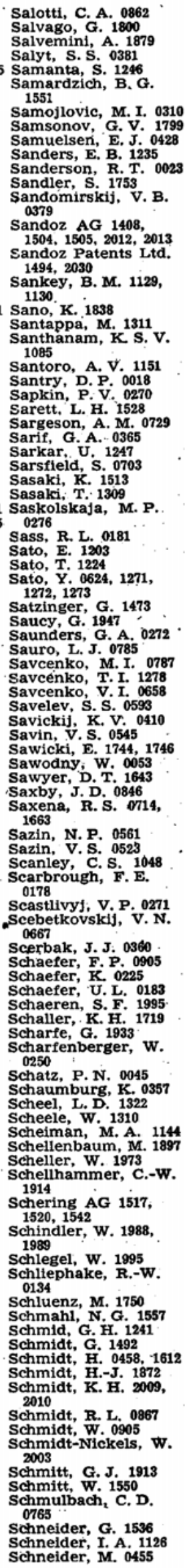 \\
\hline
\end{tabular}




\begin{tabular}{|c|c|c|c|c|c|}
\hline 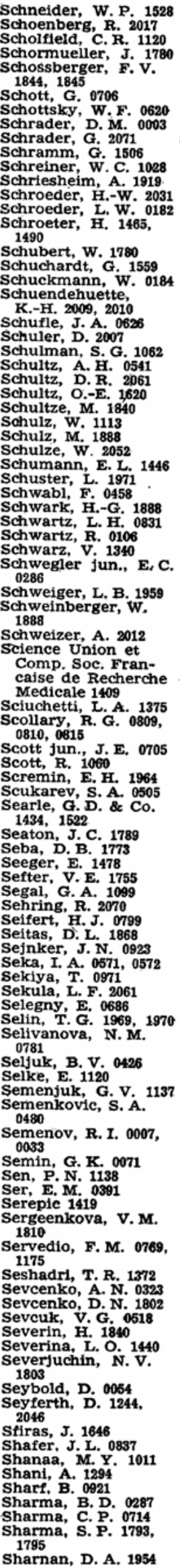 & 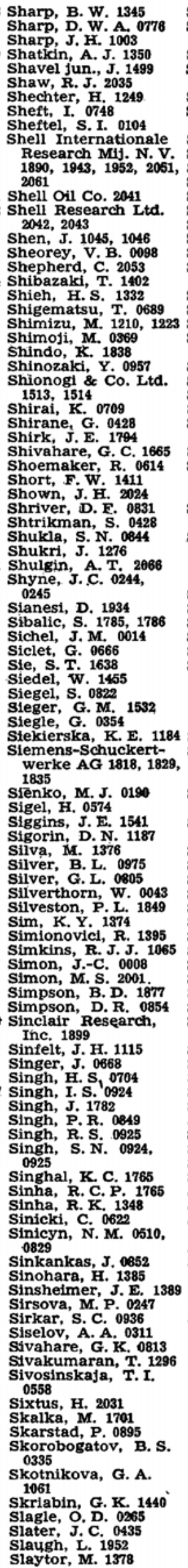 & 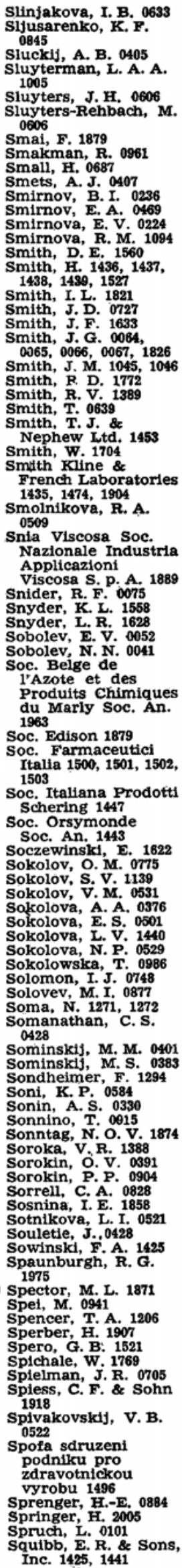 & 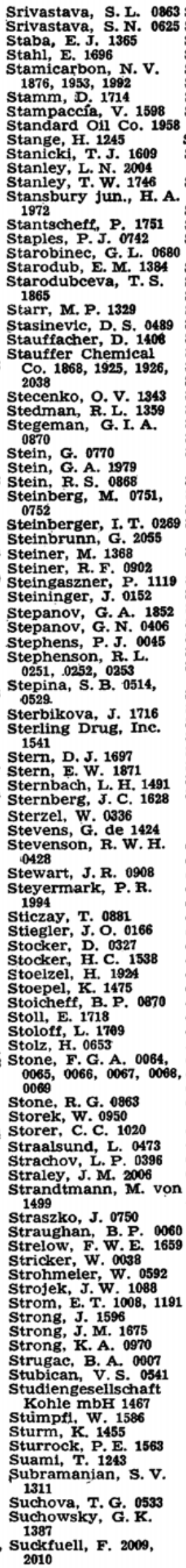 & $\begin{array}{l}\text { Suda, S. 1029 } \\
\text { Suehiro, T. } 1265 \\
\text { Suetaka, w. 0933 } \\
\text { Sugimori, S. } 1265 \\
\text { Sugiyama, N. 1266 } \\
\text { Suhl, H. 0428 } \\
\text { Sulgin, B. V. 0343 } \\
\text { Sullivan, E. P. A. } \\
\text { 0356 } \\
\text { Sulzenko, A. A. 0274 } \\
\text { Sumarokova, T. N. } \\
\text { 0796 } \\
\text { Sumitomo Chemical } \\
\text { Co. Ltd. 2036 } \\
\text { Summ, B. D. 0247 } \\
\text { Sun Oil Co. 1976 } \\
\text { Sunagawa, G. 1271, } \\
\text { 1272, 1273 } \\
\text { Supina, W. R. 1644 } \\
\text { Sur, J.S. 0444 } \\
\text { Suri, S. K. 1043 } \\
\text { Surma, D. 0983 } \\
\text { Surovo, J. N. 1805 } \\
\text { Surpina, D. E. 0796 } \\
\text { Surville, R. de 1232 } \\
\text { Sutova, G. A. 0568 } \\
\text { Suvurov, N. N. 1440 } \\
\text { Suyama, K. 1318 } \\
\text { Suzuki, H. 1152 } \\
\text { Suzuki, K. 1776 } \\
\text { Suzukd, M. 0279, 0882 } \\
\text { Svangiradze, R. R. } \\
\text { o382 }\end{array}$ & 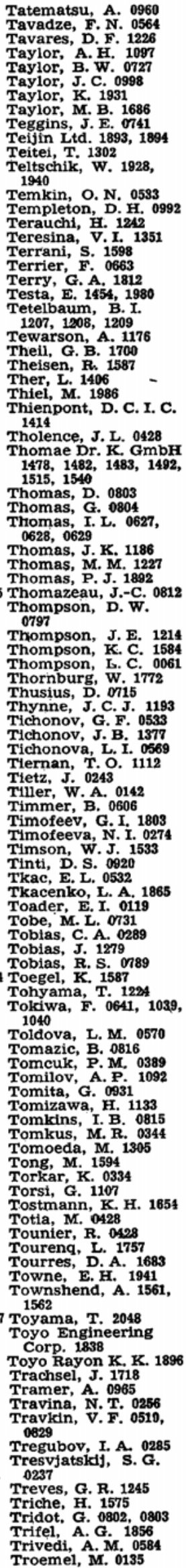 \\
\hline
\end{tabular}




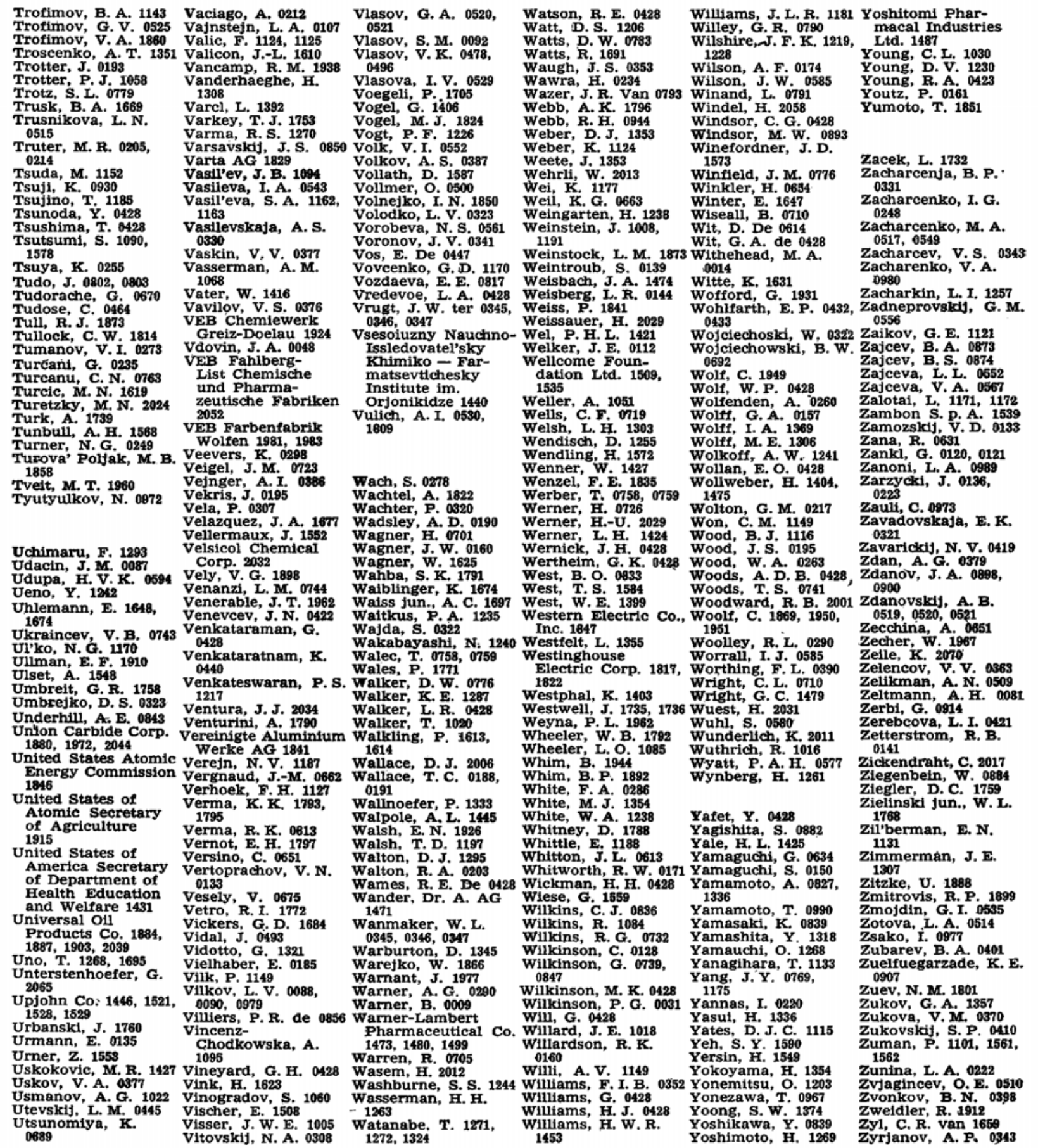

